\title{
Étude numérique d'une technique de régularisation des contraintes thermomécaniques dans les dépôts obtenus par projection plasma
}

\author{
B. Naitali ${ }^{1}$, F. Hugot ${ }^{1}$ et M. el Ganaoui ${ }^{2}$ \\ 1 Université de Limoges, École Nationale Supérieure d’Ingénieurs de Limoges, 87068 Limoges, France \\ 2 Université de Limoges, SPCTS UMR 6638 CNRS, FST, 123 Av. Albert Thomas, 87060 Limoges, France
}

Reçu le 6 september 2007

\begin{abstract}
Résumé - L'étude porte sur la modélisation thermomécanique de dépôts de zircone obtenus par projection plasma sur substrat en acier inoxydable avec une sous-couche intermédiaire en NiCrAlY. Ces dépôts sont utilisés pour la réalisation de barrières thermiques. Typiquement, un dépôt de quelques centaines de micromètres induit une diminution du niveau de température du substrat de quelques centaines de degrés suivant le flux thermique. La sous-couche de NiCrAlY permet une adaptation des valeurs des coefficients de dilatation entre le substrat et le dépôt et assure une transition favorisant ainsi l'adhérence finale du dépôt. Un modèle avec prise en compte des propriétés élastiques est développé au sein du logiciel industriel ABAQUS basé sur une approximation éléments-finis permettant une étude paramétrique de cette technique.
\end{abstract}

\begin{abstract}
This study deals with thermal and mechanical modelling of plasma sprayed zirconia coatings on stainless steel substrates, with a NiCrAlY bond coat. Applications concern thermal barrier coatings (TBC). Typically a coating of some hundreds micrometers induces a decrease of some hundreds degrees on the substrate temperature following the thermal flux. NiCrAlY permits to regularise the gap of the dilatation coefficients between the substrate and the ceramic. The NiCrAlY improves the adhesion of the coatings by providing a transition between the level of residual stresses in the three layers. A numerical model is developed and integrated to the industrial code ABAQUS based on finite elements approximation to simulates these behaviours under a parametric study.
\end{abstract}

\section{Introduction}

La projection par plasma est une technique de revêtement de surface par voie sèche qui permet de réaliser des dépôts épais (quelques dizaines à quelques centaines de micromètres) [1]. La chaleur est apportée par une source électrique. La préparation du substrat fait intervenir une opération de sablage, de manière à rendre la surface rugueuse pour favoriser l'accrochage des particules fondues. Les particules injectées sont fondues au sein du jet de plasma, s'écrasent et se solidifient sur le substrat ou sur les couches déjà déposées. Les dépôts sont composés de lamelles. La modélisation offre une voie originale pour accéder aux transferts à l'échelle des dépôts [2].

Les contraintes intervenant lors de la réalisation d'un dépôt par projection thermique ont un rôle déterminant sur les propriétés finales des pièces. Elles peuvent être à l'origine de réseaux de micro ou macro fissures, altérant les caractéristiques mécaniques du dépôt.
Les contraintes intervenant au cours du procédé de projection sont au nombre de trois :

- les contraintes superficielles dues au sablage du substrat. Cette opération généralement indispensable à l'adhésion du dépôt crée une forte compression de surface, affectant le niveau de contrainte. Pour s'affranchir des contraintes de sablage, la pièce a subi un traitement thermique de recuit de recristallisation [3] ;

- la contrainte de trempe ou de tir (Fig. 1) : les particules projetées à une température $T_{\mathrm{m}}$ subissent au contact du substrat à la température $T_{\mathrm{s}}$, ou des couches déjà déposées une trempe. Un dispositif cryogénique permet de contrôler la température pendant le tir. Leur contraction est empêchée par leur accrochage sur le substrat ce qui induit une contrainte dans le dépôt en tension :

$$
\sigma_{\mathrm{q}}=E_{\mathrm{d}} \alpha_{\mathrm{d}}\left(T_{\mathrm{m}}-T_{\mathrm{s}}\right)
$$

- la contrainte de refroidissement intervient en fin de cycle quand la pièce est refroidie jusqu'à 


\section{Nomenclature}

\begin{tabular}{|ll|}
\hline$t$ & temps \\
$E_{\mathrm{d}}$ & module de Young du dépôt \\
$\alpha_{\mathrm{d}}, \alpha_{\mathrm{s}}$ & coefficient de dilatation thermique du \\
& dépôt, du substrat \\
$T_{\mathrm{m}}$ & température des particules pendant le tir \\
$T$ & température \\
$T_{\mathrm{s}}$ & température du substrat pendant le tir \\
$T_{\text {ext }}$ & température du milieu extérieur \\
$T_{\mathrm{amb}}$ & température ambiante $\left(25^{\circ} \mathrm{C}\right)$ \\
$\sigma_{\mathrm{q}}, \sigma_{\mathrm{d}}$ & contrainte de trempe ou de tir, de refroidissement \\
$\varepsilon$ & déformation \\
$\alpha$ & diffusivité thermique \\
$h$ & coefficient d'échange superficiel de chaleur \\
$A, n$ & coefficient de fluage \\
$\nu$ & coefficient de Poisson \\
\hline
\end{tabular}
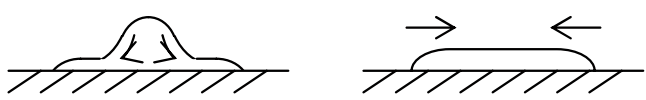

Fig. 1. Déformation particule/substrat.

la température ambiante $T_{\mathrm{amb}}$. Elle dépend du différentiel de dilatation des constituants. Dans le dépôt, la contrainte s'écrit :

$$
\sigma_{\mathrm{d}}=E_{\mathrm{d}}\left(T-T_{\mathrm{amb}}\right)\left(\alpha_{\mathrm{d}}-\alpha_{\mathrm{s}}\right)
$$

Si $\alpha_{\mathrm{d}}<\alpha_{\mathrm{s}}$, cas d'une céramique déposée sur un substrat métallique, la contrainte de refroidissement dans le dépôt est en compression.

Ces contraintes sont relaxées par micro fissuration dans les céramiques et par fluage dans les métaux.

L'objectif de ce travail est de qualifier une technique de régularisation des contraintes par voie numérique.

\section{Matériaux}

Les principales couches d'accrochage utilisées dans l'industrie sont des alliages base nickel [4]. L'accrochage est favorisé par la présence d'aluminium dans l'alliage qui a un rôle désoxydant et qui permet de réduire la porosité entre les lamelles et d'augmenter la surface de contact.

La structure étudiée comporte un substrat métallique en acier inoxydable ou aluminium, une couche d'accrochage (NiCrAlY) et un dépôt de zircone.

Les propriétés thermomécaniques des matériaux sont reportées dans le tableau 1 [5] et les coefficients de fluage dans le tableau 2.

\section{Modèles}

Les contraintes intervenant au cours du procédé de projection plasma, lors de la réalisation de dépôts
Tableau 1. Propriétés thermiques et mécaniques des matériaux.

\begin{tabular}{cccccc}
\hline Matériaux & $\begin{array}{c}E \\
(\mathrm{GPa})\end{array}$ & $\nu$ & $\begin{array}{c}\alpha \\
\left(10^{6} \mathrm{~K}^{-1}\right)\end{array}$ & $\begin{array}{c}C_{\mathrm{p}} \\
\left(\mathrm{J} \cdot \mathrm{kg}^{-1} \cdot \mathrm{K}^{-1}\right)\end{array}$ & $\begin{array}{c}\kappa \\
\left(\mathrm{W} \cdot \mathrm{m}^{-1} \cdot \mathrm{K}^{-1}\right)\end{array}$ \\
\hline Acier inox & 215 & 0,3 & 14 & 500 & 20 \\
Aluminium & 71 & 0,33 & 24 & 917 & 240 \\
$\mathrm{ZrO}_{2}$ & 200 & 0,2 & 8 & 456 & 1,5 \\
$\mathrm{NiCrAlY}$ & 205 & 0,3 & 12 & 500 & 11,6 \\
\hline
\end{tabular}

Tableau 2. Coefficient de fluage de l'acier inoxydable et $\mathrm{NiCrAlY}$

\begin{tabular}{ccc}
\hline Matériaux & $\begin{array}{c}\text { Coefficient de } \\
\text { fluage A }\end{array}$ & $\begin{array}{c}\text { Exposant de } \\
\text { fluage } n\end{array}$ \\
\hline Acier inox. & $4,23 \times 10^{-39}$ & 4 \\
NiCrAlY & $2,04 \times 10^{-34}$ & 3,35 \\
\hline
\end{tabular}

avec couche intermédiaire d'accrochage sont modélisées et intégrées au code ABAQUS en deux étapes : un calcul thermique permet d'obtenir la répartition des températures au cours de la phase de tir puis les contraintes sont obtenues via un calcul mécanique qui tient compte de l'évolution des températures précédemment calculées. Les modèles sont réalisés en géométrie axisymétrique. Les matériaux sont supposés homogènes, isotropes et leurs propriétés sont indépendantes de la température.

Des calculs préliminaires ont été réalisés afin d'optimiser le maillage et l'incrément de temps. Les contraintes relevées convergent lorsque la taille des mailles est égale à $0,3 \mu \mathrm{m}$ : c'est une densité satisfaisante entre le temps de calcul et la précision des résultats.

La résolution des équations d'évolution est effectuée avec l'aide du logiciel ABAQUS basé sur une approche de type éléments-finis. Le problème est traité en axisymétrie et en raison des symétries géométriques et matérielles, nous avons maillé seulement la moitié de l'éprouvette. Des éléments quadratiques isoparamétriques à 8 nœuds ont été utilisés, ce qui limite la nécessité d'un découpage très fin. Des conditions initiales sont appliquées aux nœuds situés sur le demi-axe longitudinal qui ne subissent aucun déplacement radial [5].

\subsection{Aspects thermiques}

Les échanges de chaleur au cours de la phase de projection sont schématisés sur la figure 2. Le substrat est préchauffé à la température de $300{ }^{\circ} \mathrm{C}$. La couche d'accrochage et le revêtement sont déposés à leur température de fusion. Pour simuler l'apport de matière pour chaque passage de la torche, des couches d'épaisseur $10 \mu \mathrm{m}$ sont déposées à chaque seconde [6-8]. Chaque couche est refroidie au contact du substrat ou des couches déjà déposées par conduction. Les échanges de chaleur s'effectuant en régime instationnaire, l'équation de la chaleur est résolue en milieu cylindrique en tenant compte des échanges par convection avec le milieu extérieur 


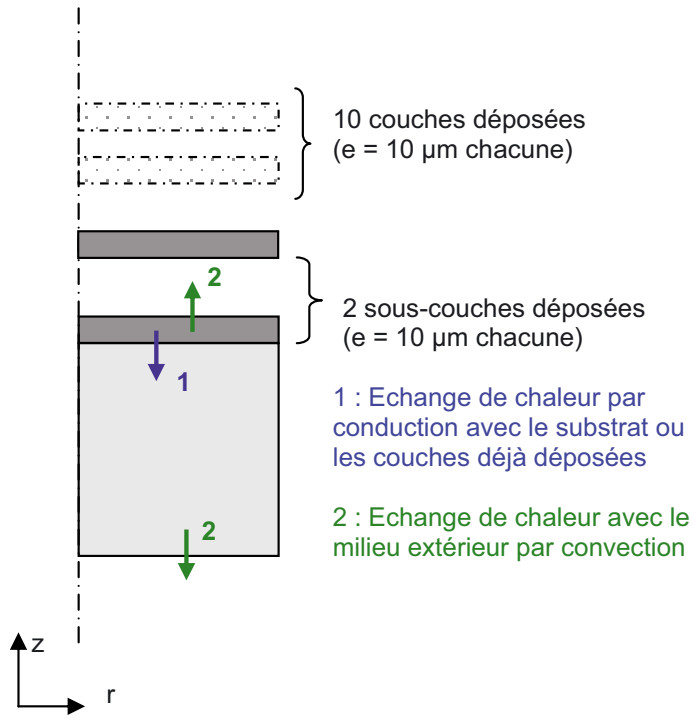

Fig. 2. Modèle thermique.

$\left(h=8 \mathrm{~W} \cdot \mathrm{m}^{-2} \cdot \mathrm{K}^{-1}\right)$. En fin de cycle, l'ensemble est refroidi lentement à la température ambiante $\left(20^{\circ} \mathrm{C}\right)$.

Le contact thermique est supposé parfait entre les couches. Le rayonnement thermique et le flux de la torche ne sont pas pris en compte.

\subsection{Aspects mécaniques}

Les contacts mécaniques entre les couches déposées sont parfaits de telle sorte qu'aucun glissement d'une couche sur une autre n'est autorisé. Deux modèles sont envisagés :

- modèle avec prise en compte des propriétés élastiques;

- modèle qui tient compte des phénomènes de relaxation des contraintes : la micro fissuration pour le dépôt de céramique et le fluage pour le substrat métallique et la sous-couche d'accrochage. La micro fissuration est modélisée en attribuant au matériau un comportement plastique idéal [8-11]. La relaxation par fluage est modélisée par la loi de Norton : $\frac{\mathrm{d} \varepsilon}{\mathrm{d} t}=A \sigma^{n}$.

\section{Résultats et discussion}

\subsection{Modèle avec couche intermédiaire}

Afin de comprendre l'influence de la sous-couche dans l'élaboration du dépôt, on se propose de comparer les contraintes intervenant au cours du procédé pour deux modèles élastiques, avec et sans couche d'accrochage.

On réalise des calculs élastiques avec les matériaux et les épaisseurs suivantes (Tab. 3).

Comme développé précédemment, les contraintes mécaniques sont obtenues via un calcul thermique préalable permettant de déterminer la répartition des températures dans les différentes couches après dépôt
Tableau 3. Épaisseur de chaque couche et conditions initiales en température.

\begin{tabular}{lccc}
\hline & Matériaux & $\begin{array}{c}\text { Épaisseur } \\
(\mathrm{mm})\end{array}$ & $\begin{array}{c}\text { Température } \\
\text { initiale }\left({ }^{\circ} \mathrm{C}\right)\end{array}$ \\
\hline Substrat & acier inoxydable & 1 & 300 \\
\hline Sous-couche & NiCrAlY & $20 \times 10^{-3}$ & 1600 \\
\hline Dépôt & zircone & $100 \times 10^{-3}$ & 2600 \\
\hline
\end{tabular}
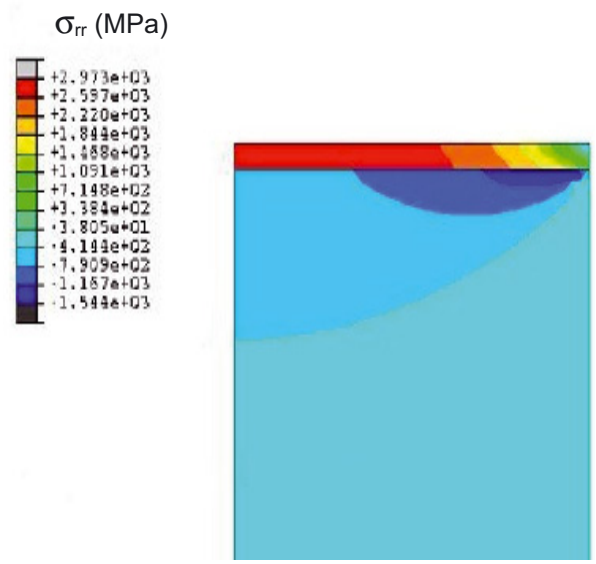

Fig. 3. Cartographie des contraintes dans la sous-couche et le substrat $(\mathrm{MPa})$ avant projection.

et avant refroidissement. Dans chaque couche, la température est quasi uniforme; elle atteint la valeur de $480{ }^{\circ} \mathrm{C}$ dans le substrat.

La figure 3 représente l'évolution de la contrainte de trempe radiale pendant le tir après le dépôt d'une souscouche de NiCrAlY d'une épaisseur de $10 \mu \mathrm{m}$. La valeur maximale de la contrainte de trempe en tension est égale à 2,9 GPa. La contrainte dans le NiCrAlY est estimée par la relation simplifiée $\sigma_{\mathrm{q}}=E_{\mathrm{d}} \alpha_{\mathrm{d}}\left(T_{\mathrm{m}}-T_{\mathrm{s}}\right)$ donnant 3,1 GPa. L'écart entre ces deux valeurs est inférieur à $10 \%$ ce qui permet de valider le modèle.

La figure 4 montre la répartition des contraintes radiales en fin de dépôt et avant refroidissement jusqu'à la température ambiante. Les contraintes relevées dans les couches déposées augmentent du substrat vers la surface extérieure du dépôt. Ceci s'explique par les mises en compression successives que subissent les couches déjà déposées sur le substrat. Ainsi la contrainte de trempe de 2,9 GPa, relevée après dépôt de la première couche de $\mathrm{NiCrAlY}$, est égale en fin de tir et avant refroidissement à $-390 \mathrm{MPa}$.

On peut accéder par simulation à l'évolution des contraintes résiduelles dans les différents matériaux, c'està-dire après refroidissement jusqu'à température ambiante $\left(20^{\circ} \mathrm{C}\right)$ du modèle.

\subsection{Comparaison des modèles avec ou sans couche d'accrochage}

Les modèles précédents montrent que les sauts de contraintes sont importants aux interfaces acier 
$\sigma_{\text {rr }}(\mathrm{MPa})$

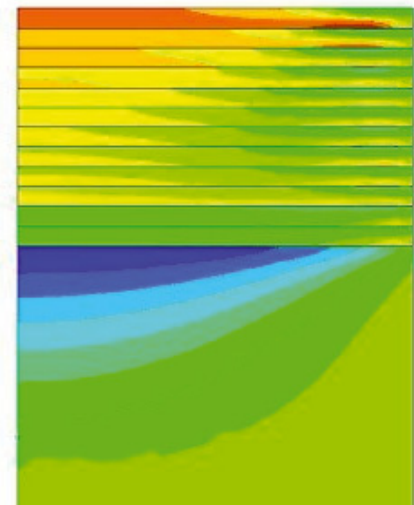

Fig. 4. Cartographie des contraintes en fin de dépôt et avant refroidissement.

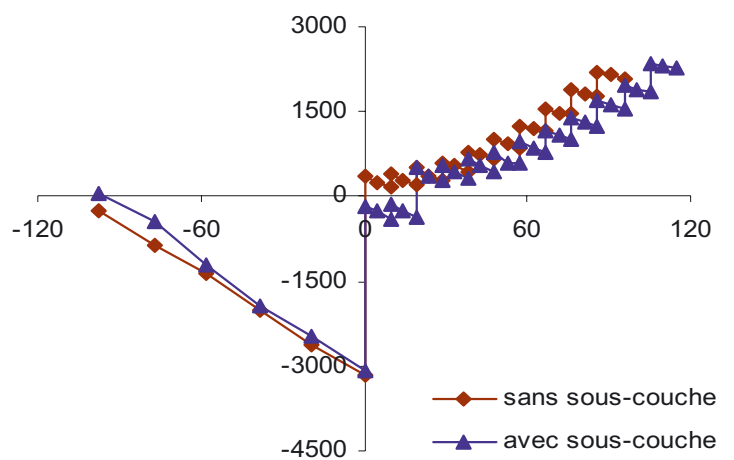

Fig. 5. Évolution de la contrainte résiduelle dans l'épaisseur du bilame avec ou sans sous-couche.

inox/NiCrAlY et NiCrAlY/zircone. Un modèle élastique équivalent mais sans couche d'accrochage a été réalisé afin de comparer les discontinuités de la contrainte résiduelle dans les deux modèles.

La figure 5 représente l'évolution des contraintes suivant l'axe de symétrie après projection pour des modèles comportant ou non une sous-couche intermédiaire en NiCrAlY.

Le saut de discontinuité des contraintes résiduelles du modèle sans couche d'accrochage est égal à 3,8 GPa, alors que pour le modèle avec sous-couche, la discontinuité maximale observée à l'interface substrat/sous-couche est inférieure à $3 \mathrm{GPa}$. De plus, le saut de contrainte à l'interface métal/céramique est nettement réduit par la présence du NiCrAlY : il est égal à $900 \mathrm{MPa}$.

La couche d'accrochage permet donc par une adaptation des coefficients de dilatation et des modules de Young de réduire un saut de discontinuité de contraintes très important par deux sauts d'amplitudes moins importantes et favoriser ainsi l'adhérence finale du dépôt.

L'adaptation des coefficients de dilatation est essentielle au cours du refroidissement final du modèle. En effet, en isolant les contraintes intervenant au cours du refroidissement, par soustraction des contraintes de trempe aux contraintes résiduelles, on obtient pour différents
Tableau 4. Contraintes en fin de refroidissement.

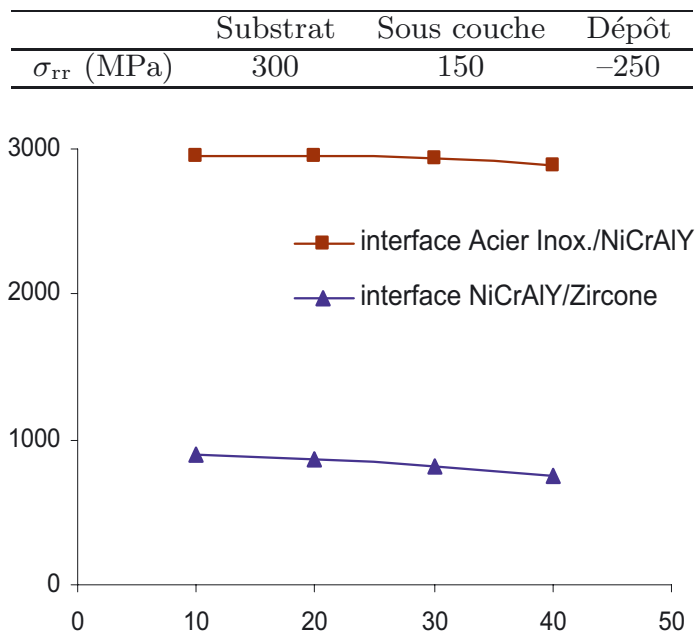

Fig. 6. Évolution de la contrainte résiduelle.

matériaux, et proche de l'interface, les résultats sont reportés dans le tableau 4.

La couche intermédiaire permet une adaptation des contraintes lors du refroidissement jusqu'à la température ambiante.

\subsection{Influence de l'épaisseur de la sous-couche}

Dans les conditions réelles d'utilisation, la sous-couche intermédiaire a une épaisseur comprise entre 10 et $50 \mu \mathrm{m}$. Sur la base du modèle élastique précédent, nous faisons varier l'épaisseur de la couche d'accrochage, par ajout successif de couches de $10 \mu \mathrm{m}$ de NiCrAlY. La figure 6 montre l'évolution des discontinuités de la contrainte résiduelle le long de l'axe de symétrie aux interfaces entre les trois matériaux intervenant en fonction de l'épaisseur de la couche de NiCrAlY.

On remarque une réduction des discontinuités de contraintes résiduelles aux interfaces, diminution plus forte pour l'interface NiCrAlY/Zircone que pour l'acier inox/NiCrAlY. L'augmentation de l'épaisseur de la couche d'accrochage - en diminuant les sauts de contraintes normales - favorise l'adhérence du dépôt.

\subsection{Influence de la nature des matériaux}

Afin d'étudier un cas extrême en terme d'écart de coefficient de dilatation, nous réalisons un modèle élastique identique au précédent, à l'exception de la nature du substrat qui est désormais en aluminium $(\alpha=24 \times$ $\left.10^{-6} \mathrm{~K}^{-1}\right)$. Les contraintes avant et après refroidissement final dans les différents matériaux sont reportées dans le tableau 5 , il s'agit des contraintes maximales au voisinage des interfaces.

Le coefficient de dilatation de l'aluminium est supérieur à celui de l'acier inoxydable donc les contraintes en compression sont très importantes dans la sous-couche 
Tableau 5. Coefficient de fluage de l'acier inoxydable.

\begin{tabular}{|c|c|c|c|c|c|c|}
\hline \multirow{2}{*}{$\begin{array}{l}\text { Nature } \\
\text { du } \\
\text { substrat }\end{array}$} & \multicolumn{3}{|c|}{$\begin{array}{c}\text { Contraintes avant } \\
\text { refroidissement }(\mathrm{MPa})\end{array}$} & \multicolumn{3}{|c|}{$\begin{array}{c}\text { Contraintes résiduelles } \\
(\mathrm{MPa})\end{array}$} \\
\hline & Substrat & NiCrAlY & Zircone & Substrat & NiCrAlY & Zircone \\
\hline Inox & -3340 & -305 & 1080 & -3200 & -91 & 684 \\
\hline $\mathrm{Al}$ & -1982 & -1142 & 975 & -1527 & -1955 & -250 \\
\hline
\end{tabular}

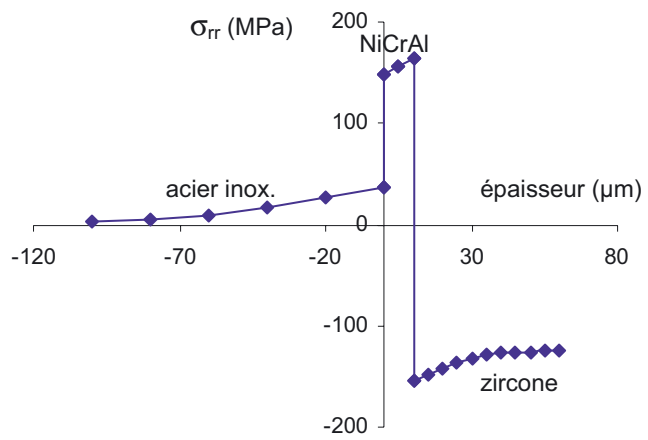

Fig. 7. Évolution de la contrainte résiduelle.

et le dépôt de zircone au cours du refroidissement. Les contraintes de refroidissement sont plus importantes avec un substrat en aluminium qu'avec l'acier inox, elle est de -1,2 GPa pour la zircone au voisinage de l'interface avec le NiCrAlY, alors que pour le substrat en acier inox, elle est de $395 \mathrm{MPa}$.

\subsection{Modélisation des phénomènes de relaxation des contraintes}

Dans le modèle qui va suivre nous prenons en compte le phénomène de fluage, pour l'acier inox et la couche de $\mathrm{NiCrAlY}$, et la micro fissuration pour la zircone. Le fluage ne sera pris en compte que pendant la phase de construction du dépôt. Ces nouvelles données entraînent des temps de calcul beaucoup plus importants et nous modélisons la structure suivante : acier inoxydable/NiCrAlY/zircone.

Les propriétés de fluage des matériaux sont indiquées dans le tableau. L'évolution des contraintes résiduelles dans le modèle est représentée sur la figure 7 .

Les contraintes relevées sont désormais de l'ordre de la centaine de MPa. Elles sont en tension dans le substrat et la couche d'accrochage, et en compression dans la céramique. Les résultats sont en bon accord avec ceux obtenus par Bengtsson et Persson [9]. En effet, leurs travaux concernent la modélisation des contraintes intervenant au cours du procédé de projection plasma lors de l'élaboration de dépôt de zircone sur des superalliages avec couche intermédiaire d'accrochage en NiCrAlY. Les contraintes résiduelles relevées sont ensuite comparées à celles obtenues avec des modèles expérimentaux. La simulation est réalisée avec le code de calcul ABAQUS. L'épaisseur de matériau déposée à chaque passage de la torche est prise en compte, et vaut $12 \mu \mathrm{m}$ pour le NiCrAlY et $13 \mu \mathrm{m}$ pour la zircone. Le substrat est préchauffé à la température de $40{ }^{\circ} \mathrm{C}$ avant le dépôt de la couche de $\mathrm{NiCrAlY}$, et à la température de $100{ }^{\circ} \mathrm{C}$ avant le dépôt de zircone. La relaxation des contraintes dans la céramique est prise en compte via un comportement parfaitement plastique. Les résultats de la modélisation s'accordent avec l'expérience. Les contraintes résiduelles dans la céramique sont en compression et de l'ordre de $-50 \mathrm{MPa}$; elles sont en tension dans la couche d'accrochage, et comprises entre 100 et $200 \mathrm{MPa}$. Le substrat au voisinage de l'interface est en tension avec des valeurs inférieures à $100 \mathrm{MPa}$.

En outre les discontinuités de contraintes relevées à chaque interface entre deux épaisseurs de $10 \mu \mathrm{m}$ d'un même matériau, pour le modèle élastique, ne sont plus rencontrées pour le modèle avec relaxation des contraintes. Enfin les contraintes de différentielles de dilatation ont désormais une contribution plus importante que les contraintes de trempe.

En cas de transition de phase, le comportement thermique peut être couplé au comportement mécanique et en particulier à la régularisation introduite par la couche du milieu $[12,13]$.

\section{Conclusion}

La couche intermédiaire joue un rôle important sur l'adhésion du dépôt. Elle permet une accommodation des valeurs des coefficients de dilatation entre substrat métallique et dépôt céramique, réduisant ainsi les discontinuités de contraintes aux interfaces. Cette adaptation des coefficients de dilatation prend toute son importance au cours du refroidissement jusqu'à la température ambiante du modèle. Les sauts de contraintes résiduelles aux interfaces entre les différents matériaux sont réduits lorsque l'épaisseur de la couche intermédiaire augmente.

Un modèle prenant en compte les phénomènes de relaxation des contraintes (micro fissuration pour la céramique et fluage pour les métaux), permet d'évaluer les contraintes résiduelles en fin de dépôt; elles sont de l'ordre de la centaine de MPa, en compression dans la céramique, et en tension dans les couches métalliques.

Dans tous les modèles réalisés, nous n'avons pas tenu compte des contraintes de sablage (quelques centaines de $\mathrm{MPa}$ ), qui provoquent des déformations plastiques sur plusieurs centaines de microns depuis la surface du substrat.

\section{Références}

[1] B. Pateyron, A. Vardelle, M. El Ganaoui, G. Delluc, P. Fauchais, 1D Modeling of coating formation under plasma 
spraying conditions : Splat cooling and laying. Progress in Plasma Processing of materials, P. Fauchais (Ed.), Begell Hoouse, 2001, pp. 519-526

[2] Kh. Fataoui, B. Pateyron, M. El Ganaoui, Simplified 2D thermo-mechanical modeling of splat formation in plasma spraying processes, International Symposium on Advances in Computational Heat Transfer, May 11-16, 2008, Marrakech, Morocco, CD ROM, Begel house, 6 p.

[3] G. De Smet, La pratique des traitements thermiques des métaux industriels, Dunod (Ed.), Paris, 1963

[4] K.A. Khor, et al. Dynamic mechanical properties of plasma sprayed Ni-based alloys, Mat. Sci. Eng. 279 (2000) 166-171

[5] S. Raghavan, H. Wang, R.B. Dinwiddie, W.D. Porter, The effect of grain size, porosity and yttria content on the thermal conductivity o nanocrystalline zirconia, Scripta Materialia 39 (1998) 1119-1125

[6] J.A. Nesbitt, Thermal modeling of various thermal barrier coatings in a high heat flux rocket engine, Surf. Coat. Technol. 130 (2000) 141-151

[7] J.F. Sacadura, Initiation aux transferts thermiques, Ed. Lavoisier Technique et Documentation, 1993
[8] J.T. Demasi, K.D. Sheffler, M. Ortiz, Thermal barrier coating life prediction model development, NASA Contractor Report 182230, 1989

[9] P. Bengtsson, C. Persson, Modelled and measured residual stresses in plasma sprayed thermal barrier coatings, Surf. Coat. Technol. 92 (1997) 78-86

[10] F. Hugot, M. Boivin, Modelisation of residual stresses generated in the course of elaboration of a silicon monofilament aluminium matrix composite, Eur. J. Mech. 9 (1990) $175-188$

[11] F. Hugot, J.C. Boyer, Modélisation du comportement thermo-élasto-plastique d'un bilame. Mise en évidence des contraintes résiduelles d'origine thermique, Eur. J. Mech. Environment 41 (1996) 131-136

[12] M. El Ganaoui, A. Lamazouade, P. Bontoux, D. Morvan, Computational solution for fluid flow under solid/liquid phase change conditions, Int. J. Comput. Fluids 31 (2002) $539-556$

[13] M. El Ganaoui, P. Bontoux, A. Lamazouade, E. Leonardi, G. de Vahl Davis, Computational model for solutal convection during directional solidification, Numer. Heat Transf. Part B 41 (2001) 325-338 